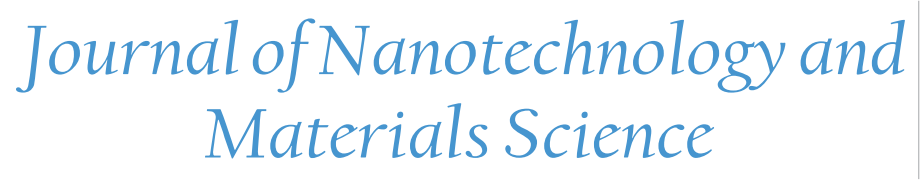

\title{
Nanotechnology and the Future of Science
}

\section{Farida Selim}

Department of Physics and Astronomy, Center for Photochemical Sciences, Bowling Green State University, Ohio, USA.

\section{Received date: December 27, 2014 Accepted date: March 16, 2015 Published date: March 18, 2015}

*Corresponding author: Selim, F. Department of Physics and Astronomy, Center for Photochemical Sciences, Bowling Green State University, Ohio, USA. E-mail: faselim@bgsu.edu

Citation: Selim, F. Nanotechnology and the Future of Science (2015) J Nanotech Mater Sci 2(1): 12

\section{Introduction}

The ability to manipulate materials at the nanometer scale and subsequent control of fundamental processes at the nanometer level have led to great improvements in the optical, electronic, magnetic, and mechanical properties of materials. Consequently nanotechnology has quickly emerged transforming all fields of science from medicine to electronic and industry ${ }^{[1]}$.

Nanotechnology addresses the ability of controlling structures, materials and devices at nanoscale level. It implies the synthesis of structures with nano size precision and develops them to systems with macrostructures and devices. Building structures and devices atom by atom would lead to the ultimate control of matter, cells, and devices. The integration of nanotechnology with the broad range of materials opens up the road to new breakthrough discoveries and inventions; it may take the human race to the ultimate goal of modifying the universe and controlling its destination ${ }^{[2]}$.

The integration of nanotechnology with a broad range of materials science dominates the topic of this issue ${ }^{[3]}$. The journal will focus on elevating fundamental understanding about the physics, chemistry and biology of nanomaterials and present significant advancement in nanosynthesis and nanotechnology. The interdisciplinary nature of novel materials and nanotechnology brings the physical, chemical and biological sciences together in strong comprehensive way. This will drive us to new great discoveries and ideas in all branches of science ${ }^{[4]}$. Similarly, this journal addresses a wide range of physics, chemistry, biology, and engineering research in matter and cells ${ }^{[5]}$.

We hope this issue will provide significant update on important topics in nanotechnology and materials science.

\section{References}

1. Drexle, E. Engines of Creation: The Coming Era of Nanotechnology. (1987) Anchor Library of Science.

2. Invernizzi, N., Foladori, G. Nanotechnology and the developing world: will nanotechnology overcome poverty or widen disparities? (2005) Nanotechnology Law \& Business 2(3): 294-303. 3. Binns, C. Introduction to Nanoscience and Nanotechnology. (2010) Wiley.

4. Aydil, E. S. Nanomaterials for Solar Cells. (2007) Nanotechnology Law \& Business 4(3): 275-291.

5. Vollath. D. Nanomaterials: An Introduction to Synthesis, Properties and Applications. (2013) Wiley. 